\title{
Rotational Magneto-Acousto-Electric Tomography (MAET): Theory and Experimental Validation
}

\author{
L. Kunyansky ${ }^{1}$, C. P. Ingram ${ }^{2}$, and R. S. Witte ${ }^{3}$ \\ ${ }^{1}$ Department of Mathematics, University of Arizona, Tucson, AZ, USA \\ ${ }^{2,3}$ Department of Medical Imaging, University of Arizona, Tucson, AZ, USA
}

May 22, 2017

\begin{abstract}
We present a novel two-dimensional (2D) MAET scanner, with a rotating object of interest and two fixed pairs of electrodes. Such an acquisition scheme, with our novel reconstruction techniques, recovers the boundaries of the regions of constant conductivity uniformly well, regardless of their orientation. We also present a general image reconstruction algorithm for the 2D MAET in a circular chamber with point-like electrodes immersed into the saline surrounding the object. An alternative linearized reconstruction procedure is developed, suitable for recovering the material interfaces (boundaries) when a non-ideal piezoelectric transducer is used for acoustic excitation. The work of the scanner and the linearized reconstruction algorithm is demonstrated using several phantoms made of high-contrast materials and a biological sample.
\end{abstract}

Keywords: Lorentz force tomography, Magneto-Acousto-Electric tomography, Electric Impedance tomography, imaging of conductivity, lead currents, synthetic transducer

\section{Introduction}

Magneto-Acousto-Electric Tomography (MAET), also known as the Lorentz force impedance tomography, is based on measurements of the electrical potential arising when an acoustic wave propagates through conductive medium placed in a magnetic field $[17,22]$. The Lorentz force resulting from the motion of free ions (and/or electrons) in the magnetic field causes separation of charges and, thus, generates Lorentz currents within the tissues. The values of electric potential associated with these currents are measured outside of the object of interest and used to reconstruct the conductivity map within the tissues.

MAET can be viewed as an attempt to significantly improve the resolution of the better known Electrical Impedance Tomography (EIT), that was introduced in the late 1980's as a fast, inexpensive, and safe method for mapping the distribution of electrical conductivity in biological tissue. In EIT, surface potentials are detected while injecting small levels of 
current through parts of the body [2-4]. A variety of medical conditions, including cancer, blood clots, and seizures, are associated with large changes in bioimpedance (see [3] and references therein). However, despite extensive development, EIT has not become a widely used technique in medical imaging due to its poor spatial resolution. EIT is based on solving an ill-posed (or unstable) inverse problem, which makes impossible obtaining high resolution images. In contrast, the stability of the inverse problem of MAET is restored by coupling electrical measurements to ultrasound waves through the Lorentz force effect. This yields high-resolution spatial information about the object, and, as a result, makes MAET a potentially irreplaceable imaging modality.

Few experimental results on MAET have been obtained by now. As a form of biomedical imaging, this technique was first introduced in [22], under the name of Hall Effect Imaging. By scanning the transducer in the plane perpendicular to its axis, the authors of [22] were able to image the interfaces between the regions of different conductivities, parallel (or nearly parallel) to the scanning plane. In [17], accurate measurements of the Lorentz force effect within a narrow measuring chamber were performed using time-harmonic ultrasound waves; image reconstruction was not attempted in that work. An image of a planar face of a simple test object was obtained in [7], again, using planar scanning parallel to that face. In [6], reconstructed images of a gelatin phantom and of a beef sample are presented. These images were also obtained using planar scanning; material interfaces parallel to the scanning plane and nearly perpendicular to the axis of the transducer are clearly visible in the images. In all of the above works, only one pair of electrodes was used, and the orientation of the transducer was stationary. This made reconstruction of the interfaces not perpendicular to the transducer axis difficult if not impossible. In the present work the scanned object is rotated with respect to the transducer, and two pairs of electrodes are used, thus permitting uniformly accurate detection of material interfaces.

In addition to MAET, there exist several other hybrid modalities utilizing various combinations of magnetic field with ultrasound, such as, for example, Magneto-Acoustic Tomography with Magnetic Induction (MAT-MI) [8, 14, 15, 24], Lorentz force electrical impedance tomography using magnetic field [25], and some others. Like MAET, all these modalities are very new; they significantly differ from the present version of MAET by the physics of the signal acquisition. For these two reasons we will not attempt here a comparative analysis of these modalities and MAET.

Like almost any other type of tomography, MAET relies on mathematical processing of the data in order to recover the desired image. One of the first rigorous image reconstruction techniques for MAET has been proposed in [20], under assumptions that the conductivity distribution $\sigma(x)$ is a small perturbation of a constant, and that the object is tested by planar time-harmonic waves of all possible frequencies and orientations. An implicit assumption in this work was that the electric potential was measured by a pair of electrodes located perpendicularly to the propagation direction of the plane wave. The technique was tested in a numerical experiment.

A significantly more general 3-dimensional (3D) setup was considered in [12]. It was assumed that at least 3 different pairs of electrodes were used (although a significant freedom was retained in modeling various electrode configurations). The magnetic field for simplicity was assumed uniform, with ability to utilize at least two perpendicular orientations of the magnetic induction. Importantly, it was assumed that the ultrasound illumination was done 
using an ideal transducer, capable of transmitting acoustic waves of all frequencies, and the scanning was done using all wave directions. It has been shown in [12] that, if such a rich set of data is available, the conductivity can be reconstructed theoretically exactly, using a set of explicit and linear formulas, without any linearization with respect to small parameters or other simplifying assumptions. Such a linearity is quite surprising, since the original problem of EIT is nonlinear, and since many other hybrid modalities lead to nonlinear inverse problems even when the coupling between the component fields is very weak. In addition, all the steps of the reconstruction procedure presented in [12] are stable. These techniques were tested in [12] in numerical experiments with simulated noisy data, confirming the theoretical conclusions of the paper.

A 2-dimensional (2D) reconstruction procedure was developed in [1] for a MAET data acquisition scheme somewhat similar to the experimental setup of [6]. As in the latter work, only one pair of wide flat electrodes was considered in [1], i.e. smaller amount of measured information is assumed, comparing to [12]. However, similarly to [12], the assumption was made that the transducer could generate all frequencies and illuminate the object from all the directions within the plane where the object was supported. (Since the experimental setup of [6] does not deliver multi-directional ultrasound excitation, methods of [1] cannot be combined directly with the data of [6].) Since in [1] only one pair of electrodes was assumed, an explicit reconstruction technique (similar to [12]) could not be applied, and a more sophisticated minimization procedure was developed. It has been shown theoretically that this procedure converges to the sought conductivity; the corresponding algorithm was validated in numerical simulations.

The reconstruction techniques of [12] and [1] share the first step consisting of reconstruction of the $\operatorname{curl}(\mathrm{s})$ of the so-called lead (or virtual) current(s) associated with each electrode pair. This step is done using any of the well known methods developed for photo- and thermoacoustic tomography (see, e.g., [9] and references therein). In order for the rest of the MAET mathematics work properly, the results of the first step should be quantitatively correct. However, in all of the recent experimental works on MAET, ultrasound waves were generated using piezoelectric transducers. Such transducers are popular in the biomedical imaging community due to their high efficiency and ability to work as both transmitters and receivers of acoustic signals. However, a significant drawback of these devices from the MAET point of view is their narrow bandwidth. A frequency response of a typical transducer can be modeled by a bell-shaped curve centered at a certain frequency (e.g., $0.5 \mathrm{MHz}$ as in several of the above mentioned experimental works), quickly falling off away from that frequency. In other words, such transducers cannot generate a significant range of lower frequency waves (say, 0 to $0.25 \mathrm{MHz}$ ). The application of the linear methods of thermoacoustic tomography to such data with missing lower frequencies, is equivalent to applying a high-pass (spatial) filter to the reconstructed function. While in some other hybrid modalities (e.g. photoacoustic tomography) such high-pass images are still useful, a quantitatively correct image needed for the multi-step MAET reconstruction procedures $[1,12]$ cannot be obtained this way.

In our opinion, MAET will be able to yield quantitatively correct, high-resolution images of conductivity if wide-band acoustic sources are used, and a sufficiently rich 3D set of data is collected. However, before an advanced 3D MAET scanner can be built, the feasibility of MAET needs to be demonstrated in lower-budget experiments utilizing conventional piezo- 
electric transducers and other readily available measuring devices. In particular, the goal of the present work is to build a prototype 2D MAET scanner and to develop theoretical and algorithmic tools for image reconstruction from the MAET data. We will show that even using a relatively simple experimental MAET scheme one can reconstruct boundaries between the regions of contrasting conductivity uniformly well, independently of their orientation. The work of the scanner will be demonstrated using tissue-mimicking phantoms and a bovine sample.

In the present paper we concentrate on the engineering aspect of MAET and limit the presentation of the underlying mathematics to the main results that are needed to understand the design and work of our scanner. A more rigorous study of the mathematical side of this work can be found in the companion paper [13].

\section{A prototype 2D MAET scanner}

To this end, the authors have built the first 2D fully-tomographic prototype MAET scanner, and tested it on several simple test objects. The purpose of this work is to show that even in a minimal configuration MAET can recover boundaries of bodies with different conductivities, with the resolution close to the wavelength corresponding to the central frequency of the transducer.

\subsection{General scheme of the experiment}

The main part of our scanner is a cylindrical scanning chamber placed between two cylindrical neodymium permanent magnets, situated coaxially above and below the chamber, and creating a near vertical magnetic induction. The chamber is filled with a saline $(\mathrm{NaCl})$ solution and the object of interest is placed inside the chamber and completely submerged into the saline. An ultrasound transducer (whose axis is horizontal) sends short pulses into the object through a side window in the chamber. The horizontal cross-section of the chamber and the top view of the data acquisition setup are shown in Figure 1.

The interaction of the magnetic field with horizontal motion of the charged particles generates Lorentz currents oriented horizontally. The secondary Ohmic currents propagate through the object and the conductive saline. Two pairs of electrodes placed in the saline near the chamber's walls pick up differential values of the resulting electric potential. The electrodes are made of vertical copper wires, and the boundaries of the test object(s) were also made vertical when possible. This results in Ohmic currents propagating mostly horizontally, and allows us to use a simplified 2D mathematical model to accurately model our experimental data and to pose the $2 \mathrm{D}$ inverse problem.

In order to obtain a sufficiently rich set of data the test object(s) is (are) suspended from a turntable rotating around the vertical axis of the chamber, and electrical measurements are repeated for different angular positions of the object. In addition, the transducer scans the object horizontally, as shown in the Figure 1. Such a scanning pattern guarantees that each segment of the object's boundary is touched tangentially by a propagating acoustic front at least once. This, in turn, stabilizes the inverse problem solved as the first step of the image reconstruction procedure, see Section 3.4. 


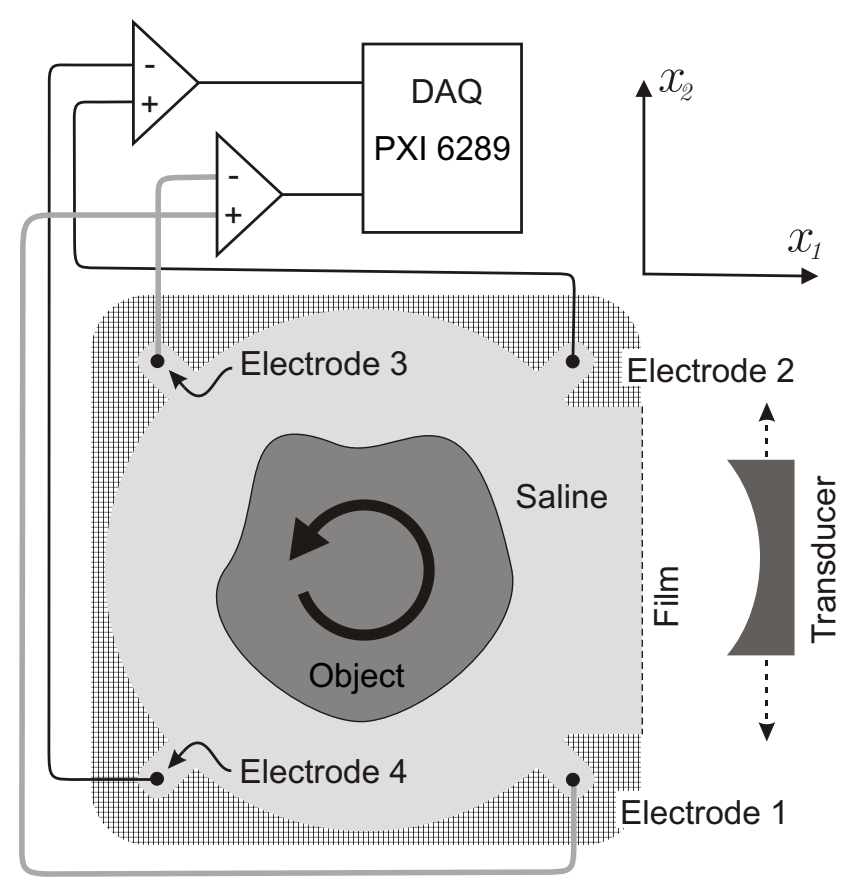

Figure 1: A propotype MAET scanner, horizontal cross-section

\subsection{Details of the data acquisition scheme}

The chamber, turntable, and spur gears driving the turntable were 3D printed of nonconductive plastic, so that the electrical currents were restricted to the near cylindrical interior of the chamber. In order to facilitate propagation of acoustic waves generated by the transducer into the chamber, both the chamber and the transducer are placed inside a larger tank filled with water. The interior of the chamber is electrically isolated from the water in the tank by a Tegaderm film covering the chamber's window. This film does not significantly interfere with the propagation of ultrasound, although a weak acoustic reflection from the film is registered by the transducer. The transducer we use has a central frequency $0.5 \mathrm{MHz}$ (Olympus Panametrics-NDT V389, $\mathrm{f}=54.6 \mathrm{~mm}$, dia $=38 \mathrm{~mm}$ ), and is driven by a rectangular pulse transmitter/receiver (Olympus Panametrics-NDT V3077PR).

The inner diameter of the chamber is $75 \mathrm{~mm}$ and the width of a window is $50 \mathrm{~mm}$. The Neodymium magnets used in the scanner are $75 \mathrm{~mm}$ in diameter and $25 \mathrm{~mm}$ tall. The vertical distance between magnets is $50 \mathrm{~mm}$; the direction of the magnetic induction is near vertical across the chamber. Measured in the center of the chamber, the magnetic induction is $0.35 \mathrm{~T}$ with gradual decrease away from the central axis of the scanner.

In our experiments, the test objects were suspended from a turntable rotated about the vertical axis of the scanner. Rotation is driven by a Velmex rotation stage, connected to the turntable through a set of spur gears. (Such a setup eliminates magnetic interaction between the electric motor and the strong field of the magnets).

The interior of the chamber is filled with a saline solution, to provide both electrical and acoustic contact with the tested object. Most of our experiments were done with $0.9 \%$ and 
$0.45 \%$ saline; stronger or weaker concentrations did not yield improvement in the signal, but both somewhat decreased the signal-to-noise ratio (SNR). A partial explanation of this phenomenon is attempted in Section 3.4.

The electric potential in saline is picked up by four copper electrodes (see Figure 1) made of straight vertical copper wires (1mm in diameter) running through the whole height of the chamber. The wires are simply the naked ends of a solid core RG59 radio-frequency cables connecting these electrodes to the amplifiers. Two high-impedance differential amplifiers (Teledyne LeCroy, 1855A) are used to capture and amplify by a factor of ten the potential difference between electrodes \#1 and \#3, and between electrodes \#2 and \#4. The two amplified signals are registered by two channels of a multifunctional DAQ card (National Instruments PXI 6289, sampling rate $20 \mathrm{Ms}$ per second).

Thus, in each experiment we collected two time sequences, $U_{1,3}(t, \theta, y)$ and $U_{2,4}(t, \theta, y)$ representing the potential differences between electrodes \#1 and \#3, and between \#2 and $\# 4$, for each position $y$ of the transducer and angular position $\theta$ of the turntable. The angle $\theta$ was sampled between 0 and 360 degrees, $x_{2}$ was scanned between -25 and $25 \mathrm{~mm}$. These two sets of data were used to reconstruct a MAET image, as described below.

\section{Mathematics of the 2D MAET}

\subsection{Electric potential}

It has been shown ([17]) that if the tissue with conductivity $\sigma(x)$ moves in a $3 \mathrm{D}$ space with velocity $\mathbf{V}(t, x)$ within the constant magnetic field $\mathbf{B}(x)$, the arising Lorentz force will generate Lorentz currents $\mathbf{J}^{L}(t, x)$ given by the following formula

$$
\mathbf{J}^{L}(t, x)=\sigma(x) \mathbf{B}(x) \times \mathbf{V}(t, x) .
$$

Throughout the paper we will make the following simplifying assumptions. The magnetic induction $\mathbf{B}$ is constant and oriented vertically, i.e. $\mathbf{B}=B \vec{e}_{3}$ (where $\vec{e}_{3}$ is the unit vector parallel to the $x_{3}$ axis, which, in turn, is perpendicular to $x_{1}$ and $x_{2}$ axes shown in Figure 1). The conductivity $\sigma(x)$ is non-zero and depends on the $2 \mathrm{D}$ variable $x=\left(x_{1}, x_{2}\right)$. The chamber walls are vertical, and the acoustic excitation is $x_{3}$-independent, with velocity $\mathbf{V}(t, x)$ oriented horizontally $^{1}$. Under these assumptions the Lorentz currents $\mathbf{J}^{L}$ and the secondary Ohmic currents $\mathbf{J}^{O}$ flow horizontally, and the mathematics of the problem becomes two-dimensional, i.e. $\mathbf{J}^{L}(t, x)=\left(J_{1}^{L}, J_{2}^{L}\right)(t, x), \mathbf{J}^{O}(t, x)=\left(J_{1}^{O}, J_{2}^{O}\right)(t, x), \mathbf{V}(t, x)=\left(V_{1}, V_{2}\right)(t, x)$, etc. Under these assumptions equation (1) takes the following form:

$$
\mathbf{J}^{L}(t, x)=\sigma(x) B \mathbf{V}^{\perp}(t, x),
$$

where $\mathbf{V}^{\perp}(t, x)$ is the left normal to $\mathbf{V}(t, x)$, i.e. $\mathbf{V}^{\perp}(t, x)=\left(-V_{2}, V_{1}\right)(t, x)$.

The Ohmic currents $\mathbf{J}^{O}(t, x)$ are related to the electric potential $u(t, x)$ in the medium by the Ohm's law

$$
\mathbf{J}^{O}=\sigma \nabla u \text {. }
$$

\footnotetext{
${ }^{1}$ If the velocity field is not strictly horizontal, then, due to the properties of the cross product in equation (1), the vertical component of the velocity will have no effect on $\mathbf{J}^{L}$ — if $\mathbf{B}$ is strictly vertical, as we assume. If $\mathbf{B}$ is also not strictly vertical, there will be some blurring of the boundaries in the image.
} 


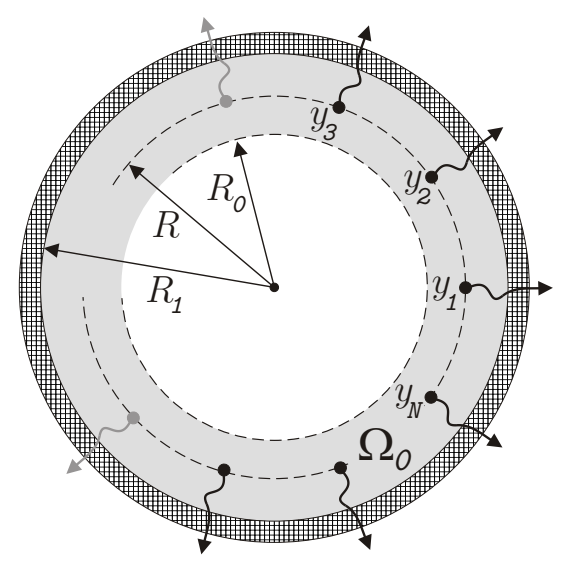

Figure 2: An idealized MAET chamber

Since the propagation of charges is divergence-free and $\operatorname{div}\left(\mathbf{J}^{L}+\mathbf{J}^{O}\right)=0$, we obtain

$$
\nabla \cdot \sigma \nabla u=-\nabla \cdot \mathbf{J}^{L} .
$$

Let us consider an acquisition scheme that involves a circular chamber and a set of $N$ equispaced electrodes. (Our actual set-up is a particular case of this more general scheme, with $N=4$.) We assume that the interior of the chamber is a disk of radius $R_{1}$ as shown in Figure 2 where the means of delivering ultrasound excitation are omitted. The $N$ electrodes are placed at the points $y_{j}$ lying on the concentric circle of radius $R$ in an equispaced fashion:

$$
y_{j}=R\left(\cos \psi_{j}, \sin \psi_{j}\right), \quad \psi_{j}=\Psi+\frac{2 \pi(j-1)}{N}, \quad j=1, \ldots, N,
$$

where $\Psi$ angle determines the angular position of the first detector. We will denote the disk (of radius $R_{1}$ ) describing the interior of the chamber by $\Omega$, and its boundary by $\partial \Omega$. The object of interest is contained within a smaller concentric circle of radius $R_{0}$; the interior of the latter circle will be denoted by $\Omega_{0}$. The ring $\Omega \backslash \Omega_{0}$ is filled by the saline with constant conductivity $\sigma_{0}$.

The chamber walls are non-conductive, therefore, there are no currents through $\partial \Omega$ and the normal component of the total current $\mathbf{J}^{L}(t, x)+\mathbf{J}^{O}(t, x)$ vanishes on $\partial \Omega$ :

$$
\sigma_{0} \frac{\partial}{\partial n} u(t, z)=-\mathbf{J}^{L} \cdot n(z), \quad z \in \partial \Omega,
$$

where $n(z)$ is the exterior normal to $\partial \Omega$ at point $z$.

We assume that the speed of sound $c$ in the tissues and the density $\rho$ of the tissues are constant and coincide with those of the surrounding saline. The pressure of the sound waves $p(t, x)$ satisfies the standard linear wave equation:

$$
\frac{1}{c^{2}} \frac{\partial^{2}}{\partial t^{2}} p(t, x)=\Delta p(t, x), \quad x \in \mathbb{R}^{2} .
$$


Additionally, $p(t, x)$ is the time derivative of the velocity potential $\varphi(t, x)$ (see, for example [5]), so that

$$
\mathbf{V}(t, x)=\frac{1}{\rho} \nabla \varphi(t, x), \quad p(t, x)=\frac{\partial}{\partial t} \varphi(t, x) .
$$

The above formulas show that not only the components of the model velocity satisfy the wave equations, but that the velocity itself is a gradient vector field. This property needs to be taken into account when modeling the acoustic fields of a transducer; otherwise, the total model of MAET measurements may give non-physical results.

\subsection{Lead currents}

The measurements of the electric potentials $u\left(t, y_{j}\right)$ made by point-like electrodes at the points $y_{j} \in \Omega_{0}$, (see equation (4)) are combined with weights $W_{j}$ to obtain a measurement functional $M(t, \mathbf{W})$ :

$$
M(t, \mathbf{W})=\sum_{j=1}^{N} W_{j} u\left(t, y_{j}\right), \quad \mathbf{W}=\left(W_{1}, \ldots, W_{N}\right) .
$$

From the engineering standpoint, if $N$ is even, this can be accomplished by combining several standard differential measurements. In our scanner, for example, we measure the voltage differentials $u\left(t, y_{1}\right)-u\left(t, y_{3}\right)$ and $u\left(t, y_{2}\right)-u\left(t, y_{4}\right)$, out of which various combinations in the form (7) can be generated.

The only restriction we impose on the choice of weights $W_{j}$ is that their sum should equal to 0 :

$$
\sum_{j=1}^{N} W_{j}=0
$$

This requirement is needed, in part, because potential $u(t, x)$ is defined only up to an arbitrary constant. If (8) is satisfied, measured values $M(t, \mathbf{W})$ (equation (7)) do not depend on the choice of this constant. The simplest example of such a measurement is a standard differential measurement with $N=2, W_{1}=1$ and $W_{2}=-1$.

As it is usually done when investigating MAET, we introduce the notion of the lead (or virtual) current. This is the current that would propagate through the chamber (containing the saline and the object of interest) if one injected currents $W_{j}$ through the electrodes. Quantitatively, the density and direction of the lead current also describes the sensitivity of the system of electrodes to a unit electrical dipole placed at the varying locations within the chamber, if the potential recorded on each electrode is weighed with a weight $W_{j}$. As a result, the MAET measurements can be expressed in terms of the lead currents, as explained below.

Let us consider an auxiliary problem of finding the electric potential $w(x)$ within $\Omega$ in the absence of Lorentz currents, but with currents injected in the medium through the point electrodes placed at the same points $y_{j}$ as defined above, with currents $W_{j}$ injected through the electrodes placed at $y_{j}, j=1, \ldots, N$. As explained in the Appendix, such a lead potential $w_{\mathbf{W}}(x)$ satisfies the conductivity equation in $\Omega$ with punched out points $y_{j}, j=1, \ldots, N$ 
(where it becomes singular)

$$
\nabla \cdot \sigma(x) \nabla w_{\mathbf{W}}(x)=0, \quad x \in \Omega \backslash \bigcup_{j=1}^{N} y_{j} ;
$$

it also satisfies the Neumann boundary condition on the regular (non-conductive) boundary

$$
\frac{\partial}{\partial n} w_{\mathbf{w}}(z)=0, \quad z \in \partial \Omega
$$

and the asymptotic growth condition at the singular points

$$
w_{\mathbf{W}}(x)=\frac{1}{2 \pi \sigma_{0}} W_{j} \ln \left|x-y_{j}\right|+\mathcal{O}(1) \text { as } x \rightarrow y_{j}, \quad j=1, \ldots, N .
$$

(The big $\mathcal{O}$ notation in the last equation indicates that the difference between $w_{\mathbf{W}}(x)$ and $\frac{1}{2 \pi \sigma_{0}} W_{j} \ln \left|x-y_{j}\right|$ remains bounded in the limit $\left.x \rightarrow y_{j}\right)$.

Solution $w_{\mathbf{W}}$ of the equation (9) with boundary conditions (10) and (11) can be found as the sum of two functions $w_{\mathbf{W}}^{\text {sing }}(x)$ and $w_{\mathbf{W}}^{\text {smooth }}(x)$,

$$
w_{\mathbf{W}}(x)=w_{\mathbf{W}}^{\text {sing }}(x)+w_{\mathbf{W}}^{\text {smooth }}(x),
$$

where $w_{\mathbf{W}}^{\text {sing }}$ is defined by the following formula

$$
w_{\mathbf{W}}^{\operatorname{sing}}(x) \equiv \frac{1}{2 \pi \sigma_{0}} \sum_{j=1}^{N} W_{j} \ln \left|x-y_{j}\right|,
$$

and $w_{\mathbf{W}}^{\text {smooth }}(x)$ is found as the solution of the following boundary value problem in $\Omega$ :

$$
\begin{aligned}
\nabla \cdot \sigma \nabla w_{\mathbf{W}}^{\text {smooth }}(x) & =-\chi(x) \nabla \cdot \sigma(x) \nabla w_{\mathbf{W}}^{\text {sing }}(x), \quad x \in \Omega . \\
\frac{\partial}{\partial n} w_{\mathbf{W}}^{\text {smooth }}(z) & =-\frac{\partial}{\partial n} w_{\mathbf{W}}^{\text {sing }}(z), \quad z \in \partial \Omega,
\end{aligned}
$$

where the indicator function $\chi(x)$ is defined as as follows

$$
\chi(x)=\left\{\begin{array}{l}
0, \quad x \in \Omega_{0}, \\
1, \quad x \in \Omega \backslash \Omega_{0} .
\end{array}\right.
$$

Equation (14) with boundary conditions (15) has a unique solution if condition (8) is satisfied[13]. It is easy to see that, since $w_{\mathbf{W}}^{\text {smooth }}$ is bounded in $\Omega$, the sum (12) satisfies equations (9) and (10) and has the desired behavior (equation (11)) at the singular points.

The lead current $\mathbf{J}^{\mathbf{W}, \sigma}$ (corresponding to a particular choice of weights $\mathbf{W}$ and given $\sigma(x)$ ) is now defined in $\Omega$ through the lead potential $w_{\mathbf{W}}$ as follows

$$
\mathbf{J}^{\mathbf{W}, \sigma}(x)=\left(J_{1}^{\mathbf{W}, \sigma}, J_{2}^{\mathbf{W}, \sigma}\right)(x)=\sigma(x) \nabla w_{\mathbf{W}}(x) .
$$

Below we will have to deal with the $2 \mathrm{D} \operatorname{curl} C^{\mathbf{W}, \sigma}(x)$ of this current defined as

$$
C^{\mathbf{W}, \sigma}(x) \equiv \frac{\partial}{\partial x_{1}} J_{2}^{\mathbf{W}, \sigma}(x)-\frac{\partial}{\partial x_{2}} J_{1}^{\mathbf{W}, \sigma}(x)
$$


The lead current analyzed above can be physically realized by applying a set of voltages to the point-like electrodes. In addition to such currents we will need to analyze currents that would be excited in our medium by an external potential $w^{\text {in }}$ that would exist in a medium with uniform conductivity. Such a potential can be represented by a function harmonic in $\Omega$. We thus consider the following problem: given a function $w^{\text {in }}(x)$ harmonic in $\Omega$, find the solution $w^{\text {out }}$ to the following boundary value problem

$$
\begin{aligned}
\nabla \cdot \sigma \nabla w^{\text {out }}(x) & =-\chi(x) \nabla \cdot \sigma(x) \nabla w^{\text {in }}(x), \quad x \in \Omega, \\
\frac{\partial}{\partial n} w^{\text {out }}(z) & =0, \quad z \in \partial \Omega .
\end{aligned}
$$

It is shown in [13] that this problem has a unique solution $w^{\text {out }}$, and the sum $w^{\text {in }}+w^{\text {out }}$ solves the conductivity equation in $\Omega$ :

$$
\nabla \cdot \sigma(x) \nabla\left[w^{\text {in }}(x)+w^{\text {out }}(x)\right]=0, \quad x \in \Omega .
$$

Thus, we define a virtual current $\mathbf{J}^{w^{\text {in }}, \sigma}$ induced by the potential $w^{\text {in }}$ in $\Omega$ filled with the medium with conductivity $\sigma(x)$, by the formula

$$
\mathbf{J}^{w^{\text {in }}, \sigma}(x)=\left(J_{1}^{w^{\text {in }}, \sigma}, J_{2}^{w^{\text {in }}, \sigma}\right)(x) \equiv \sigma(x) \nabla\left[w^{\text {in }}(x)+w^{\text {out }}(x)\right] .
$$

We, in particular, are interested in the $2 \mathrm{D} \operatorname{curl} C^{w^{\mathrm{in}}, \sigma}(x)$ of this current defined as follows

$$
C^{w^{\mathrm{in},}, \sigma}(x)=\frac{\partial}{\partial x_{1}} J_{2}^{w^{\mathrm{in}}, \sigma}(x)-\frac{\partial}{\partial x_{2}} J_{1}^{w^{\mathrm{in}}, \sigma}(x) .
$$

We notice that both curls $C^{\mathbf{W}, \sigma}$ and $C^{w^{\text {in }}, \sigma}$ are finitely supported within $\Omega \backslash \Omega_{0}$ and vanish within $\Omega_{0}$, since $\sigma(x)=\sigma_{0}$ in $\Omega_{0}$.

Finally, by substituting (19) into (20) we obtain for future reference, the following equation:

$$
C^{w^{\text {in }}, \sigma}=\frac{\partial \sigma}{\partial x_{1}} \frac{\partial}{\partial x_{2}}\left[w^{\text {in }}+w^{\text {out }}\right]-\frac{\partial \sigma}{\partial x_{2}} \frac{\partial}{\partial x_{1}}\left[w^{\text {in }}+w^{\text {out }}\right]=J_{2}^{w^{\text {in }}, \sigma} \frac{\partial \ln \sigma}{\partial x_{1}}-J_{1}^{w^{\text {in }}, \sigma} \frac{\partial \ln \sigma}{\partial x_{2}}
$$

\subsection{Lead currents and MAET measurements}

The lead current $\mathbf{J}^{\mathbf{W}}$ (given by (16)) plays an important role in the analysis of the MAET measurements $M(t, \mathbf{W})$. It can be shown ([13]) that

$$
M(t, \mathbf{W})=-B \int_{\Omega} \mathbf{J}^{\mathbf{W}} \cdot \mathbf{V}^{\perp}(t, x) d x
$$

The above equation (22) shows that the weighted measurements $M(t, \mathbf{W})$ can be expressed through the magnetic induction and velocity of the medium physically present in the system, and through the lead currents that are not. This seeming contradiction is easily explained: the lead current describes the sensitivity of our measuring system to the Lorentz potential (2) within the medium. 
Let us recall that our velocity field is given by (6); using integration by parts one obtains

$$
\begin{aligned}
M(t, \mathbf{W}) & =-\frac{B}{\rho} \int_{\Omega}\left(\frac{\partial \varphi}{\partial x_{1}} J_{2}^{\mathbf{W}}-\frac{\partial \varphi}{\partial x_{2}} J_{1}^{\mathbf{W}}\right) d x \\
& =\frac{B}{\rho} \int_{\Omega} \varphi(t, x) C^{\mathbf{W}, \sigma}(x) d x+\frac{B}{\rho} \int_{\partial \Omega} \varphi(t, z)\left[n_{2}(z) J_{1}^{\mathbf{W}}(z)-n_{1}(z) J_{2}^{\mathbf{W}}(z)\right] d z,
\end{aligned}
$$

where $n(z)=\left(n_{1}, n_{2}\right)(z)$ is the exterior normal to $\partial \Omega$. In many important situations the above equation can be further simplified. For example, if the object is illuminated by ultrasound pulses (as is done in our scanner), there is a time interval during which velocity potential $\varphi(t, x)$ is supported strictly inside $\Omega$ (i.e., it vanishes on the boundary $\partial \Omega$ ). If $t$ lies within this time interval, (23) simplifies to

$$
M(t, \mathbf{W})=\frac{B}{\rho} \int_{\Omega} \varphi(t, x) C^{\mathbf{W}, \sigma}(x) d x .
$$

\subsection{Basic properties of MAET measurements}

It is easy to check that within any region $\Omega_{c} \subset \Omega$ in which $\sigma(x)$ is constant, the lead current $\mathbf{J}^{\mathbf{W}, \sigma}$ is curl-free, i.e. $C^{\mathbf{W}, \sigma}=0$ in $\Omega_{c}$. Indeed, similarly to (21) one obtains

$$
\begin{aligned}
C^{\mathbf{W}, \sigma} & =\frac{\partial}{\partial x_{1}}\left(\sigma(x) \frac{\partial}{\partial x_{2}} w_{\mathbf{W}}(x)\right)-\frac{\partial}{\partial x_{2}}\left(\sigma(x) \frac{\partial}{\partial x_{1}} w_{\mathbf{W}}(x)\right) \\
& =\frac{\partial w_{\mathbf{W}}(x)}{\partial x_{2}} \frac{\partial \sigma(x)}{\partial x_{1}}-\frac{\partial w_{\mathbf{W}}(x)}{\partial x_{1}} \frac{\partial \sigma(x)}{\partial x_{2}}=J_{2}^{\mathbf{W}, \sigma} \frac{\partial \ln \sigma}{\partial x_{1}}-J_{2}^{\mathbf{W}, \sigma} \frac{\partial \ln \sigma}{\partial x_{2}} .
\end{aligned}
$$

If $\sigma(x)$ is constant, in the above equation the partial derivatives containing $\sigma(x)$ vanish, yielding $C^{\mathbf{W}, \sigma}=0$. Therefore it follows from equation (24) that at any time $t$ when the ultrasound pulse is supported strictly within $\Omega_{c}$, the MAET signal $M(t, \mathbf{W})$ is equal to zero. In other words, there is no MAET signal from regions of constant conductivity; if the object consists of such regions, the signal will be generated only when the pulse propagates through the boundary between these regions. Nevertheless, if a sufficient amount of information is acquired, the conductivity can, in theory, be reconstructed exactly at each point in $\Omega$, from MAET measurements (see $[1,12]$ and the explanation given below).

Another interesting observation has implications to modeling and testing MAET. Suppose $w(x)$ is a lead potential satisfying equation (9) with boundary conditions (10) and (11), and with given conductivity $\sigma(x)$. Then the same equations are also satisfied by a lead potential $w_{1}(x)=C w(x)$ with conductivity $\sigma_{1}(x)=\frac{1}{C} \sigma(x)$, where $C$ is an arbitrary non-zero

factor. The lead current $\mathbf{J}_{1}(x)=\sigma_{1}(x) \nabla w_{1}(x)=\frac{\sigma(x)}{C} \nabla[C w(x)]=\mathbf{J}(x)$ clearly remains the same. Therefore, MAET measurements with $\sigma(x)$ replaced by $\sigma_{1}(x)$ will remain unchanged, according to (22). There is no contradiction between this fact and our ability to reconstruct $\sigma(x)$ from MAET measurements, since it is assumed that we know the values of $\sigma(x)$ on the boundary $\partial \Omega$. This property also explains, at least partially, why we obtain approximately the same strength of the signal and the SNR, when using saline with different concentrations 
of $\mathrm{NaCl}$ in the range of, say, from $0.3 \%$ to $2 \%$. (However, one has to keep in mind that the currents depend on the conductivity non-linearly, and a more quantitative analysis of this phenomenon is far beyond the scope of the present paper.)

If one assumes that a sufficiently rich set of acoustic illuminations can be applied to the stationary object while doing MAET measurements (e.g., the object can be illuminated from all the directions and the bandwidth of the acoustic signal is infinite), then the curl $C^{\mathbf{W}, \sigma}$ in (24) can be reconstructed exactly. Indeed, such an ideal acoustic illumination implies that an arbitrary set of functions $\varphi(t, x)$ can be formed either directly, or by combining the measurements corresponding to several different propagating waves (so called synthetic focusing, see [10] and [11]). For example, one can focus $\varphi(t, x)$ to approximate at $t=t_{0}$ a Dirac delta-function $\varphi\left(t_{0}, x\right)=\delta(x-y)$; then equation $(24)$ yields value $C^{\mathbf{W}, \sigma}(y)$ (up to a known factor $\left.\frac{B}{\rho}\right)$. Alternatively, one can generate monochromatic plane waves of different frequencies and directions, thus recovering the Fourier transform of $C^{\mathbf{W}, \sigma}$. This procedure is described in [12], and is alluded to in [1]. After $C^{\mathbf{W}, \sigma}$ is recovered at each point of the domain, one can recover the corresponding lead current. In the case when two or more lead currents are measured, one can use such currents as a local basis and reconstruct the gradient of $\ln \sigma$, and thus the conductivity $\sigma$ ([12]). If only one current is measured, the problem can be solved by the optimization procedure developed in [1].

The above reconstruction procedures are not directly applicable to the present MAET scanner. First, in order to obtain a multi-directional acoustic illumination the object is rotated, while the electrodes remain stationary. Therefore, at each position of the turntable a new lead current is present, and the synthetic focusing in the form assumed in the previous works cannot be applied. Moreover, the use of piezoelectric transducers leads to a loss of significant part of low-frequency information about the curl $C^{\mathbf{W}, \sigma}$. Thus, the lead current(s) cannot be accurately reconstructed (even if all illumination directions were utilized), invalidating the known methods of reconstruction. Below we develop exact and approximate reconstruction techniques that can be used for processing the real data we have.

\section{MAET with a rotating object}

In this section we describe reconstruction techniques that can be used with our MAET scanner where the object is rotated, the electrodes are stationary, and the transducer does not emit lower frequencies.

\subsection{Synthetic flat transducer}

In spite of the frequent use of focusing transducers in ultrasound imaging, for a given transducer the precise space- and time-dependent velocity field of an acoustic wave in a liquid is not easy to obtain. Direct application of our MAET techniques, however, would require such an information, since the direction of Lorentz currents is closely related to the velocity of the wave in a given point. We circumvent this obstacle by utilizing a synthetic flat transducer, as follows. For a given angular position of the object, we average electric measurements for all transversal positions of the transducer (i.e. we average in $x_{2}$, see Figure 1). Since 
our measurements depend on the velocity potential $\varphi(t, x)$ linearly, the averaged values we obtain are equal to the electric response to a field produced by a very wide flat transducer.

In order to formulate a mathematical model for the corresponding $\varphi(t, x)$ we take into account that the (relatively small) vertical components of the velocity do not produce Lorentz currents ( $\mathbf{V}$ is perpendicular to $\mathbf{B}$ ), and that the transducer is activated by a very short unipolar electric pulse that can be approximated by the Dirac's delta function in time. The resulting acoustic wave can be modeled as a short plain wave propagating in the $x_{1}$ direction away from the transducer.

Within the present section (Section 4) we assume for simplicity that our synthetic transducer has an ideal frequency response. A more realistic, band limited (with absent low frequencies) model is considered in Section 5). Thus, our present model for $\varphi(t, x)$ has the following form

$$
\varphi(t, x)=C_{\operatorname{tran}} \delta\left(-x_{\operatorname{tran}}+x_{1}+c t\right),
$$

where $\delta$ is the Dirac's delta function, $C_{\text {tran }}$ is a constant depending on the transducer that we assume to be known, and $x_{\text {tran }}$ is the $x_{1}$ coordinate of the transducer. From a physical stand point the latter formula implies a flat frequency response (for $\varphi$ as a function of excitation), and yields the following expressions for the velocity and pressure

$$
\begin{aligned}
p(t, x) & =C_{\operatorname{tran}} c \delta^{\prime}\left(-x_{\operatorname{tran}}+x_{1}+c t\right) \\
\mathbf{V}(t, x) & =\frac{C_{\text {tran }}}{\rho} \vec{e}_{1} \delta^{\prime}\left(-x_{\operatorname{tran}}+x_{1}+c t\right) .
\end{aligned}
$$

The above formula for $\mathbf{V}(t, x)$ yields values that integrate to 0 in $t$, which properly represents the fact that the working surface of the transducer returns to the initial position after the pulse. We notice that formulas (25), (26), and (27) cannot be valid outside of the range of positions of the transducer in $x_{2}$ variable; however, we will only need these approximations to be valid inside the region $\Omega \backslash \Omega_{0}$, where the curl of a lead current is not zero.

By combining equations (24).and (25) we obtain

$$
\begin{aligned}
M(t, \mathbf{W}) & =\frac{B C_{\operatorname{tran}}}{\rho} \int_{\Omega} \delta\left(-x_{\operatorname{tran}}+x_{1}+c t\right) C^{\mathbf{W}, \sigma}(x) d x \\
& =\frac{B C_{\operatorname{tran}}}{\rho} \int_{\mathbb{R}} C^{\mathbf{W}, \sigma}\left[\left(x_{\operatorname{tran}}-c t\right) \vec{e}_{1}+s \vec{e}_{2}\right] d s,
\end{aligned}
$$

where we, for convenience, extended $C^{\mathbf{W}, \sigma}$ by 0 to $\mathbb{R}^{2}$. The latter formula represents a set of integrals of $C^{\mathbf{W}, \sigma}$ over a family of vertical lines; this set can be viewed as a Radon projection of $C^{\mathbf{W}, \sigma}$. In the next section we briefly review basic properties of the Radon transform.

\subsection{Basic facts about the Radon transform in $2 \mathrm{D}$}

Suppose a function $f(x)$ is finitely supported within a disk $D$ of radius $R_{0}$. The Radon transform $\mathcal{R} f$ is the values of line integrals of $f$ over all straight lines:

$$
g(p, \omega) \equiv(\mathcal{R} f)(p, \omega)=\int_{\mathbb{R}} f\left(p \omega+s \omega^{\perp}\right) d s, \quad p \in \mathbb{R}, \quad \omega \in \mathbb{S}^{1}
$$


where $\omega^{\perp}$ is the left unit normal to $\omega$. (For convenience we extended the definition by zero to the lines that do not intersect the support of $f$.)

The Radon transform can be inverted, i.e. $f$ can be reconstructed from projections $g$. We will do this using the well-known filtration backprojection algorithm (see, e.g., [18]) consisting of applying the ramp filter to $g$ :

$$
g_{M}(p, \omega) \equiv \frac{1}{2 \pi} \int_{\mathbb{R}}|\rho|\left[\int_{\mathbb{R}} g(p, \omega) h(x) e^{-i p \cdot \rho} d p\right] e^{i x \cdot \rho} d \rho,
$$

and back-projecting the filtered projections $g_{M}(p, \omega)$ :

$$
f(x)=\int_{\mathbb{S}^{1}} g_{M}(x \cdot \omega, \omega) d \omega, \quad x \in \mathbb{R}^{2} .
$$

It follows from formula (28) that

$$
\left(\mathcal{R} C^{\mathbf{W}, \sigma}\right)\left(p, \vec{e}_{1}\right)=\frac{\rho}{B C_{\operatorname{tran}}} M\left(\frac{x_{\operatorname{tran}}-p}{c}, \mathbf{W}\right),
$$

i.e. from one set of electric measurements one can recover one Radon projection (corresponding to $\omega=\vec{e}_{1}$ ) of curl $C^{\mathbf{W}, \sigma}$. In order to recover a full set of projections of a function describing an object, one usually rotates a detector with respect to the object, or the object with respect to the stationary detector. In our case, the function we would like to reconstruct represents the curl $C^{\mathbf{W}, \sigma}$ of a lead current. If we turn the object but leave weights W unchanged, the new current will not be a rotation of the original one. We address this problem in the next section.

\subsection{Synthetically rotating the currents}

Our circular domain $\Omega$ with the fixed set of electrodes at points $y_{j}$ (see Figure 2 and equation (4)) is invariant with respect to rotations by any angle $\theta_{k}=2 \pi k / N, k \in \mathbb{Z}$. This means that if a lead current is generated by a set of weights $W$, then by rotating the object by the angle $\theta_{k}$ and by properly re-assigning weights, one will rotate the original current by $\theta_{k}$. By doing this with $k=0, \ldots, N-1$ one could obtain a total of $N$ projections of $C^{\mathbf{W}, \sigma}$. However, the number of projections needed for a high resolution reconstruction is usually measured in hundreds; it would be impractical to have so many electrodes. Therefore, a more sophisticated approximate technique is developed below.

Let us consider virtual current and the corresponding 2D curl induced by the excitation potential $w^{\text {in }}$, determined by formulas (17), (18), (19), and (20). We will utilize the current $\mathbf{J}^{\gamma, \sigma}$ and curl $C^{\gamma, \sigma}$ that correspond to the linear excitation in the form

$$
w^{\text {in }}(x)=w^{\gamma}(x) \equiv \beta x \cdot \gamma
$$

where $\gamma=(\cos \alpha, \sin \alpha)$ is a given unit vector, and $\beta$ is a constant to be defined below. We notice that such current may not be easy to physically obtain in our system, since $w^{\gamma}(x)$ 
does not satisfy the zero Neumann boundary conditions. However, as we discuss below, $C^{\gamma, \sigma}$ can be approximated inside $\Omega \backslash \Omega_{0}$ by a lead current corresponding to a certain combination of weights $\mathbf{W}$. Moreover, if the object is rotated, one can also excite a rotated version of the current $C^{\gamma, \sigma}$ by changing the weights $\mathbf{W}$.

In order to explain this idea in detail, consider a linear operator $\mathfrak{R}_{\varphi}$ on $\mathbb{R}^{2}$ that rotates a vector clockwise by the angle $\varphi$. Then the curl $C^{\mathfrak{R}_{-\varphi} \gamma, \sigma\left(\Re_{\varphi} x\right)}(x)$ corresponding to the rotated conductivity $\sigma\left(\mathfrak{R}_{\varphi} x\right)$ and the rotated potential

$$
w^{\gamma}\left(\mathfrak{R}_{\varphi} x\right) \equiv \beta \mathfrak{R}_{\varphi} x \cdot \gamma=\beta x \cdot \mathfrak{R}_{-\varphi} \gamma
$$

is the counterclockwise rotation of the original current $C^{\gamma, \sigma}$ :

$$
C^{\mathfrak{R}_{-\varphi} \gamma, \sigma\left(\Re_{\varphi} x\right)}(x)=C^{\gamma, \sigma(x)}\left(\Re_{\varphi} x\right) .
$$

Let us now consider a set of weights $\mathbf{W}^{\gamma}=\left(W_{1}^{\gamma}, \ldots, W_{N}^{\gamma}\right)$ subordinated to vector $\gamma$ and given by the following formula:

$$
W_{j}^{\gamma} \equiv \frac{1}{N R} y_{j} \cdot \gamma=\frac{1}{N} \cos \left(\frac{2 \pi(j-1)}{N}+\Psi-\alpha\right) .
$$

Correspondingly, for the rotated $\gamma$ the weights $\mathbf{W}^{\mathfrak{R}_{-\varphi} \gamma}=\left(W_{1}^{\mathfrak{R}_{-\varphi} \gamma}, \ldots, W_{N}^{\mathfrak{R}_{-\varphi} \gamma}\right)$ will have values

$$
W_{j}^{\Re_{-\varphi} \gamma} \equiv \frac{1}{N R} y_{j} \cdot \Re_{-\varphi} \gamma=\frac{1}{N} \cos \left(\frac{2 \pi(j-1)}{N}+\Psi-\alpha+\varphi\right) .
$$

As shown in the companion paper [13], the resulting function $w_{\mathfrak{R}_{-\varphi \gamma}}^{\text {sing }}$ (see equation (13)) approximates within $\Omega \backslash \Omega_{0}$ the linear potential $w^{\gamma}\left(\Re_{\varphi} x\right)$ (equation(31)). Further, the resulting $2 \mathrm{D}$ curl $C^{\mathbf{W}^{\Re}-\varphi^{\gamma, \sigma}}$ of the lead current $\mathbf{J}^{\mathbf{W}^{\mathfrak{R}_{-} \gamma, \sigma}}$ (as defined by the equations (13), (12), (14), (15), and (16)) approximates the curl $C^{\gamma, \sigma(x)}\left(\mathfrak{R}_{\varphi} x\right)$ excited by potential $w^{\gamma}\left(\mathfrak{R}_{\varphi} x\right)$ :

$$
C^{\mathbf{W}^{\Re}-\varphi, \sigma}(x) \approx C^{\gamma, \sigma(x)}\left(\Re_{\varphi} x\right), \quad x \in \Omega \backslash \Omega_{0} .
$$

Such approximations become more accurate if the number of electrodes $N$ is increased, or the ratio $R / R_{0}$ becomes large. For fixed values of these parameters a certain error is introduced when $C^{\mathbf{W}^{\mathfrak{R}-\varphi^{\gamma, \sigma}}}(x)$ is used instead of $C^{\gamma, \sigma(x)}\left(\mathfrak{R}_{\varphi} x\right)$. From the practical point of view, such an error was acceptable in our experiments. The technique of rotating currents synthetically, as presented above, allows us to obtain the Radon projections of the rotated current without rotating the electrodes physically.

Thus, if we measure the corresponding acoustic response $M\left(t, \mathbf{W}^{\mathfrak{R}_{-\varphi} \gamma}\right)$ given by equation (28)

$$
\begin{aligned}
M\left(t, \mathbf{W}^{\mathfrak{R}_{-\varphi} \gamma}\right) & =\frac{B C_{\operatorname{tran}}}{\rho} \int_{\mathbb{R}} C^{\mathbf{W}^{\mathfrak{R}_{-\varphi} \gamma, \sigma}}\left(\left(x_{\operatorname{tran}}-c t\right) \vec{e}_{1}+s \vec{e}_{2}\right) d s \\
& \approx \frac{B C_{\operatorname{tran}}}{\rho} \int_{\mathbb{R}} C^{\gamma, \sigma(x)}\left(\left(x_{\operatorname{tran}}-c t\right) \mathfrak{R}_{\varphi} \vec{e}_{1}+s \mathfrak{R}_{\varphi} \vec{e}_{2}\right) d s \\
& =\frac{B C_{\operatorname{tran}}}{\rho}\left(\mathcal{R} C^{\gamma, \sigma(x)}\right)\left(\left(x_{\operatorname{tran}}-c t\right), \mathfrak{R}_{\varphi} \vec{e}_{1}\right),
\end{aligned}
$$


then the Radon projections $g(p, \omega)$ of $C^{\gamma, \sigma(x)}$ can be approximately computed from $M$ as follows

$$
g\left(p, \Re_{\varphi} \vec{e}_{1}\right) \equiv\left(\mathcal{R} C^{\gamma, \sigma(x)}\right)\left(p, \Re_{\varphi} \vec{e}_{1}\right) \approx \frac{\rho}{B C_{\text {tran }}} M\left(\frac{x_{\text {tran }}-p}{c}, \mathbf{W}^{\mathfrak{R}_{-\varphi} \gamma}\right) .
$$

Now, if the measurements are done for all values of $\varphi$ in the interval $[0,2 \pi]$, curl $C^{\gamma, \sigma(x)} \operatorname{can}$ be reconstructed from $g(p, \omega)$ by using the Radon inversion formula (equations (29), (30)). This represents the first step of the reconstruction procedure in [1] or [12] allowing one to find the conductivity by using one of the algorithms proposed in the above mentioned papers.

\subsection{Summary of the algorithm}

To summarize, this reconstruction procedure involves the following steps:

- Select two perpendicular directions $\gamma^{(1)}\left(\alpha_{1}\right)$ and $\gamma^{(2)}\left(\alpha_{2}\right)$ with $\alpha_{2}=\alpha_{1}+\pi / 2$; choose the number of object's angular positions $N$, and set $\delta \varphi=2 \pi / N$.

- For each $j=1, \ldots, N$ :

1. rotate the object to the position $\varphi_{j}=j \delta \varphi$;

2. form vectors $\mathbf{W}^{\mathfrak{R}_{-\varphi_{j}} \gamma^{(1)}}$ and $\mathbf{W}^{\mathfrak{R}_{-\varphi_{j}} \gamma^{(2)}}$ with components given by equation (33) with $\varphi=\varphi_{j}$ and $\alpha=\alpha_{1}, \alpha_{2}$;

3. measure $M\left(\left(t, \mathbf{W}^{\mathfrak{R}_{-} \varphi_{j}} \gamma^{(1)}\right)\right.$ and $M\left(t, \mathbf{W}^{\mathfrak{R}_{-\varphi_{j}} \gamma^{(2)}}\right)$ by averaging all measurements made by scanning the transducer in $x_{2}$ direction;

4. compute $g^{(1)}\left(p, \mathfrak{R}_{\varphi_{j}} \vec{e}_{1}\right)$ and $g^{(2)}\left(p, \mathfrak{R}_{\varphi_{j}} \vec{e}_{1}\right)$ by applying formula (34) to $M\left(t, \mathbf{W}^{\mathfrak{R}_{-\varphi_{j}} \gamma^{(1)}}\right)$ and $M\left(t, \mathbf{W}^{\Re-\varphi_{j}} \gamma^{(2)}\right)$.

- Reconstruct curls $C^{\gamma^{(1)}, \sigma}$ and $C^{\gamma^{(2)}, \sigma}$ by applying the inverse Radon transform (equations (29), (30)) to the data $g^{(1)}\left(p, \mathfrak{R}_{\varphi_{j}} \vec{e}_{1}\right), \quad j=1, . ., N$, and $g^{(2)}\left(p, \mathfrak{R}_{\varphi_{j}} \vec{e}_{1}\right), j=1, . ., N$.

- Reconstruct lead currents $\mathbf{J}^{\gamma^{(1)}, \sigma}$ and $\mathbf{J}^{\gamma^{(1)}, \sigma}$, and the conductivity $\sigma(x)$ from $C^{\gamma^{(1)}, \sigma}$ and $C^{\gamma^{(2)}, \sigma}$ by following either procedure presented in [12] or the algorithm of [1].

\section{Linearized reconstruction}

The reconstruction procedures developed in the previous sections and elsewhere, are all based on the assumption that the acoustic excitations are rich enough to allow for the quantitatively accurate reconstruction of the $\operatorname{curl}(\mathrm{s})$ of the lead current(s). However, piezoelectric transducers commonly utilized in practical implementations of MAET cannot emit a wide range of lower frequencies. This disallows the use of the above mentioned methods. In the present section we develop a rather crude approximate reconstruction technique that recovers boundary of the objects from the band-limited measurements delivered by the real scanner we have built. 
Our scanner has four electrodes located at the points $y_{j}=R\left(\cos \psi_{j}, \sin \psi_{j}\right), \psi_{j}=\Psi+\frac{\pi}{2} j$, $\Psi=-\frac{\pi}{4}, j=1, \ldots, 4$. We consider two vectors, $\gamma^{(m)}=\left(\cos \alpha_{m}, \sin \alpha_{m}\right), m=1,2$, with $\alpha_{1}=-\pi / 4$ and $\alpha_{2}=\pi / 4$, and seek to reconstruct (as a first step) $2 \mathrm{D}$ curls $C^{\gamma^{(j)}, \sigma}$ of the virtual currents excited by linear potentials $w^{\text {in, }(m)}(x)=w^{\gamma^{(m)}}(x) \equiv \beta x \cdot \gamma^{(m)}, m=1,2$. By applying formula (33) with such parameters, one obtains the following values for weights $\mathbf{W}^{\mathfrak{R}_{-\varphi} \gamma^{(1)}}$ and $\mathbf{W}^{\mathfrak{R}_{-\varphi} \gamma^{(2)}}$ :

$$
\mathbf{W}^{\mathfrak{R}_{-\varphi} \gamma^{(1)}}=\frac{1}{4}(\cos \varphi,-\sin \varphi,-\cos \varphi, \sin \varphi), \quad \mathbf{W}^{\mathfrak{R}_{-\varphi} \gamma^{(2)}}=\frac{1}{4}(\sin \varphi, \cos \varphi,-\sin \varphi,-\cos \varphi) .
$$

Equation (34) with the above choices of $\mathbf{W}^{\mathfrak{R}_{-\varphi} \gamma^{(1)}}$ and $\mathbf{W}^{\mathfrak{R}_{-\varphi} \gamma^{(2)}}$ yields approximate Radon projections, and the filtration backprojection algorithm $((29)$ and (30)) then yields (approximately) curls $C^{\gamma^{(1)}, \sigma}$ and $C^{\gamma^{(2)}, \sigma}$.

We would like to have a reconstruction technique that (unlike [12]) does not require explicit reconstruction of the lead currents. Let us consider a situation where the conductivity is a slight perturbation of a constant, i.e.

$$
\sigma(x)=\sigma_{0}+\varepsilon \sigma_{1}(x)
$$

Recall that curl $C^{\gamma^{(m)}, \sigma}$ corresponds to the solution of the problem (17), (18) with the right hand side $w^{\text {in }}$ equal to the linear potential $\beta x \cdot \gamma^{(m)}$. If $\varepsilon=0$ then corresponding $w^{\text {out }}=0$ and current $\mathbf{J}^{\gamma^{(m)}, \sigma_{0}}(x)$ is a constant vector:

$$
\mathbf{J}^{\gamma^{(m)}, \sigma_{0}}(x)=\sigma_{0} \nabla\left(\beta x \cdot \gamma^{(m)}\right)=\sigma_{0} \beta \gamma^{(m)}, \quad m=1,2 .
$$

Then, for $\varepsilon>0$,

$$
\mathbf{J}^{\gamma^{(m)}, \sigma}(x)=\sigma_{0} \beta \gamma^{(m)}+O(\varepsilon) \approx \sigma_{0} \beta \gamma^{(m)}, \quad m=1,2
$$

By applying formula $(21)$ to $C^{\gamma^{(m)}, \sigma}(x)$ we obtain

$$
C^{\gamma^{(m)}, \sigma}(x)=J_{2}^{w^{\text {in }}, \sigma}(x) \frac{\partial \ln \sigma(x)}{\partial x_{1}}-J_{1}^{w^{\text {in }}, \sigma}(x) \frac{\partial \ln \sigma(x)}{\partial x_{2}}, \quad m=1,2 .
$$

By combining (37) and (38) we relate $C^{\gamma^{(m)}, \sigma}$ to directional derivatives of $\ln \sigma$ :

$$
C^{\gamma^{(m)}, \sigma}(x) \approx \sigma_{0} \beta\left[\gamma_{2}^{(m)} \frac{\partial \ln \sigma(x)}{\partial x_{1}}-\gamma_{1}^{(m)} \frac{\partial \ln \sigma(x)}{\partial x_{2}}\right], \quad m=1,2 .
$$

We have chosen vectors $\gamma^{(m)}$ so that $\gamma^{(2)}$ is the right normal to $\gamma^{(1)}$, i.e. $\gamma_{1}^{(2)}=-\gamma_{2}^{(1)}$ and $\gamma_{2}^{(2)}=\gamma_{1}^{(1)}$. Taking this into account yields

$$
\begin{aligned}
& C^{\gamma^{(1)}, \sigma}(x) \approx-\sigma_{0} \beta \gamma^{(1)} \cdot \nabla \ln \sigma(x)=-\sigma_{0} \beta \frac{\partial \ln \sigma(x)}{\partial \gamma^{(1)}} \\
& C^{\gamma^{(2)}, \sigma}(x) \approx \sigma_{0} \beta \gamma^{(2)} \cdot \nabla \ln \sigma(x)=\sigma_{0} \beta \frac{\partial \ln \sigma(x)}{\partial \gamma^{(2)}}
\end{aligned}
$$


By computing directional derivatives of the last two equations one can find the Laplacian of $\ln \sigma:$

$$
\frac{1}{\sigma_{0} \beta}\left(\frac{\partial}{\partial \gamma^{(2)}} C^{\gamma^{(2)}, \sigma}(x)-\frac{\partial}{\partial \gamma^{(1)}} C^{\gamma^{(1)}, \sigma}(x)\right) \approx \frac{\partial^{2} \ln \sigma(x)}{\left(\partial \gamma^{(1)}\right)^{2}}+\frac{\partial^{2} \ln \sigma(x)}{\left(\partial \gamma^{(2)}\right)^{2}}=\Delta \ln \sigma(x) .
$$

In other words, under the assumptions that the conductivity is close to a constant, and that the transducer is ideal, equation (39) reconstructs the Laplacian of the conductivity logarithm $\Delta \ln \sigma$. The advantage of this procedure in comparison with the existing, more accurate MAET reconstruction techniques, is that it still can be applied when the transducer is significantly band-limited, since it foregoes the reconstruction of the lead currents. Moreover, it is quite easy to understand what exactly is reconstructed in the band-limited case. Indeed, if the transducers response is not ideal, equation (25) should be replaced by

$$
\varphi(t, x)=C_{\text {tran }} \eta\left(-x_{\text {tran }}+x_{1}+c t\right)
$$

where the 1D Fourier transform of $\hat{\eta}(\rho)$ is the frequency response of the transducer. This in turn, results in measuring the convolution $g(p, \omega) * \eta(p)$ in the first variable, instead of $g(p, \omega)$ given by the equation (34). It is well known, that when the filtration/backprojection formula is applied to projections of a function convolved with a given function $\eta$, the result of reconstruction is represented by the convolution of the true reconstruction with a function $\Xi(x)$ whose $2 \mathrm{D}$ transform equals to $\hat{\eta}(|\xi|)$. In other words, instead of $C^{\gamma^{(m)}, \sigma}$ we obtain the convolution of $C^{\gamma^{(m)}, \sigma}(x)$ with the function $\Xi(x)$. Further, the derivatives in equation (39) commute with convolutions. Therefore, instead of $\Delta \ln \sigma(x)$ our method will reconstruct the convolution $(\Delta \ln \sigma(x)) * \Xi(x)$. In particular, if the transducer does not reproduce lower frequencies, the reconstructed image will represent a high-frequency version of $\Delta \ln \sigma(x)$ resulting in a further emphasis of the boundaries, and amplification of oscillations in the image.

\subsection{Summary of the algorithm}

To summarize, the linearized algorithm involves the following steps:

- Select two perpendicular directions $\gamma^{(1)}\left(\alpha_{1}\right)$ and $\gamma^{(2)}\left(\alpha_{2}\right)$ with $\alpha_{1}=-\pi / 4$ and $\alpha_{2}=$ $\pi / 4$; choose the number of object's angular positions $N$, and set $\delta \varphi=2 \pi / N$.

- For each $j=1, \ldots N$ :

1. rotate the object to the position $\varphi_{j}=j \delta \varphi$;

2. form vectors $\mathbf{W}^{\mathfrak{R}_{-\varphi_{j}} \gamma^{(1)}}$ and $\mathbf{W}^{\mathfrak{R}_{-\varphi_{j}} \gamma^{(2)}}$ with components given by equation (35) with $\varphi=\varphi_{j}$;

3. measure $M\left(\left(t, \mathbf{W}^{\Re \varphi_{j}} \gamma^{(1)}\right)\right.$ and $M\left(t, \mathbf{W}^{\Re_{-\varphi_{j}} \gamma^{(2)}}\right)$ by averaging all measurements made by scanning the transducer in $x_{2}$ direction;

4. compute $g^{(1)}\left(p, \mathfrak{R}_{\varphi_{j}} \vec{e}_{1}\right)$ and $g^{(2)}\left(p, \mathfrak{R}_{\varphi_{j}} \vec{e}_{1}\right)$ by applying formula (34) to $M\left(t, \mathbf{W}^{\mathfrak{R}_{-\varphi_{j}} \gamma^{(1)}}\right)$ and $M\left(t, \mathbf{W}^{\Re_{-\varphi_{j}}} \gamma^{(2)}\right)$. 


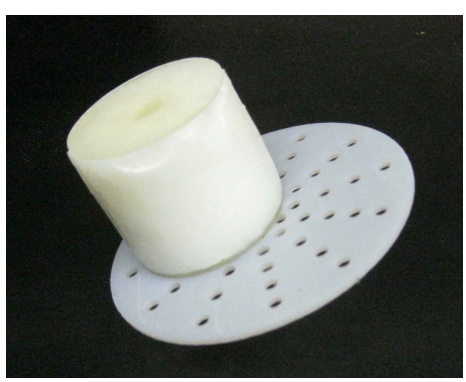

(a)

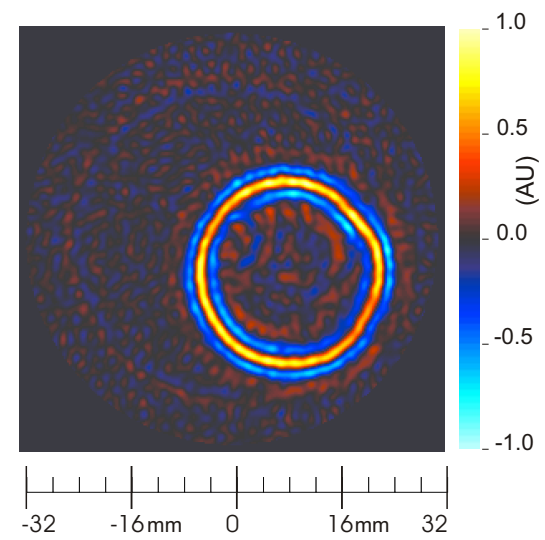

(b)

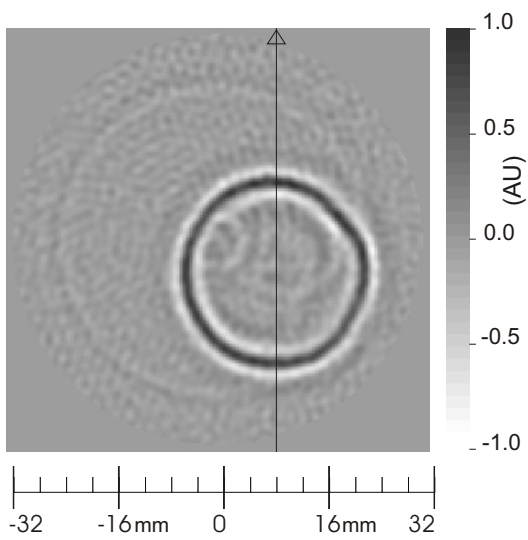

(c)

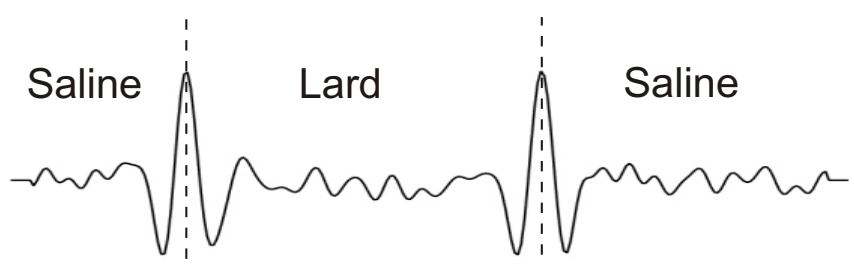

(d)

Figure 3: First test object: a lard cylinder (a) phantom attached to the turntable (b) color map of the reconstructed image $-\Delta \ln \sigma * \Xi(x)$ (c) grey scale image of the image (d) intensity profile of the image along the vertical line shown in part (c)

- Reconstruct curls $C^{\gamma^{(1)}, \sigma}$ and $C^{\gamma^{(2)}, \sigma}$ by applying the inverse Radon transform (equations (29), (30)) to the data $g^{(1)}\left(p, \mathfrak{R}_{\varphi_{j}} \vec{e}_{1}\right), j=1, . ., N$, and $g^{(2)}\left(p, \mathfrak{R}_{\varphi_{j}} \vec{e}_{1}\right), j=1, . ., N$.

- Reconstruct an approximation to $\Delta \ln \sigma(x)$ from $C^{\gamma^{(1)}, \sigma}$ and $C^{\gamma^{(2)}, \sigma}$ using formula (39).

(The reader is reminded that, if the transducer is band-limited, instead of $\Delta \ln \sigma(x)$ our method will reconstruct the convolution $\Delta \ln \sigma(x) * \Xi(x)$.)

\section{Examples of reconstruction}

In this section we demonstrate performance of our scanner and the reconstruction algorithm of Section 5 in several experiments involving some high-contrast phantoms and a real biological object. In all of the experiments the number of the Radon projections (i.e. angular positions of the object) was 200. The number of steps in the transducer's movement in the transversal direction was 40 for each projection. In order to increase the SNR of the signal, each measurement was averaged several hundred times (256 to 1024, depending on the experiment). The pulse repetition rate was $1 \mathrm{KHz}$. Depending on the amount of averaging, the total scan would take from approximately an hour to two and a half hours. 


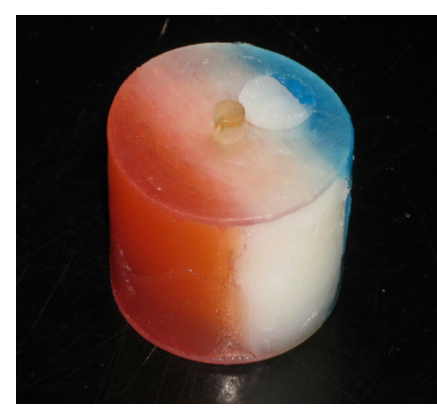

(a)

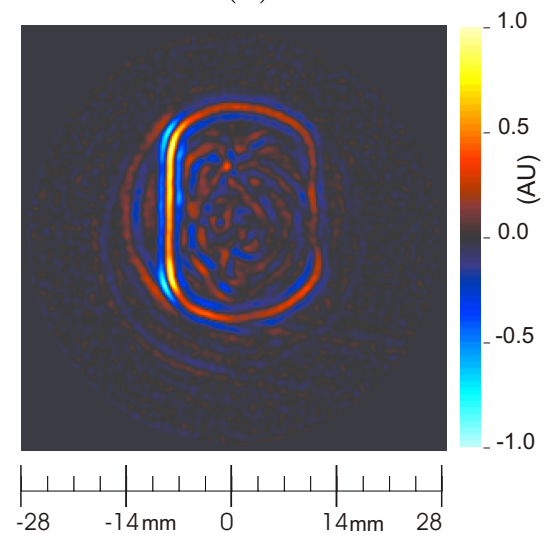

(c)

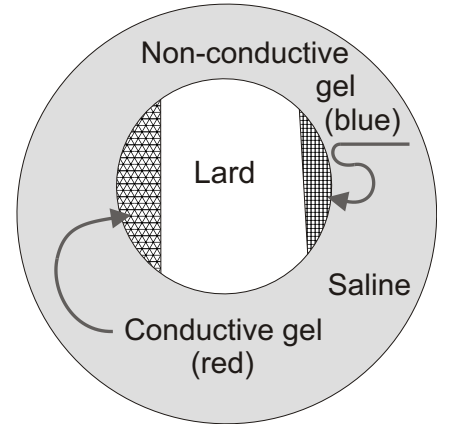

(b)

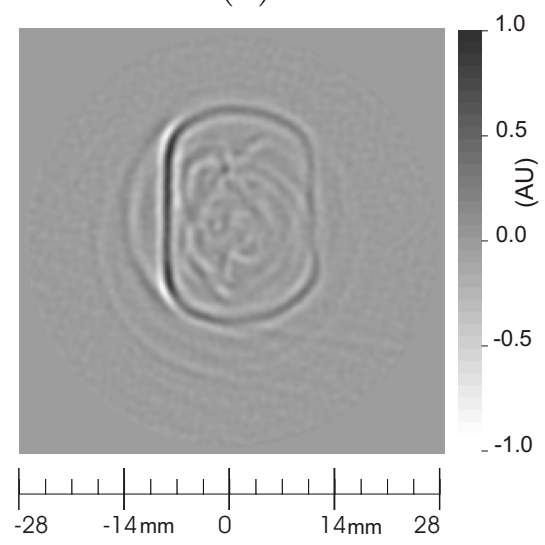

(d)

Figure 4: Reconstruction of a phantom consisting of layers of lard, red gel $(3 \% \mathrm{NaCl})$ and blue gel $(0 \% \mathrm{NaCl})$ (a) the phantom (b) inner structure of the object and its positioning on the holder (c) reconstruction shown using color scale (d) grey scale view of the reconstructed image

Our phantoms were made to have a significant contrast of conductivities, and had vertical boundaries to adhere to the two-dimensional nature of the measurements. All the test objects were immersed in a $0.9 \%$ saline solution.

In general, we do not expect such a prolonged immersion in saline to have significant effect on conductivity of tissues in biomedical applications, since we use the physiological concentration $(0.9 \%)$ that naturally occurs in human blood. However, we believe it did have an adverse effect on those of our test objects that were made of agarose gel (see the discussion of the "layered phantom" below). This, however, is a difficulty facing the experimenters, and not a drawback of the method.

The measured signal was pre-processed by applying a band pass filter $\eta(\xi)$ in the frequency domain. The filter was a product of two function, $\eta(\xi)=\eta_{1}(\xi) \eta_{2}(\xi)$ with

$$
\eta_{1}(\xi)=\left\{\begin{array}{cl}
0.5\left(1-\cos \left(\pi \xi / \xi_{1}\right)\right), & |\xi| \leq \xi_{1} \\
1, & |\xi|>\xi_{1}
\end{array}, \quad \eta_{2}(\xi)=\left\{\begin{array}{cc}
\left.\cos \left(0.5 \pi \xi / \xi_{2}\right)\right), & |\xi| \leq \xi_{2} \\
0, & |\xi|>\xi_{2}
\end{array},\right.\right.
$$

where the typical values of the cut-off frequency $\xi_{2}$ and parameter $\xi_{2}$ were $0.85 \mathrm{MHz}$ and $0.3 \mathrm{MHz}$, respectively. 


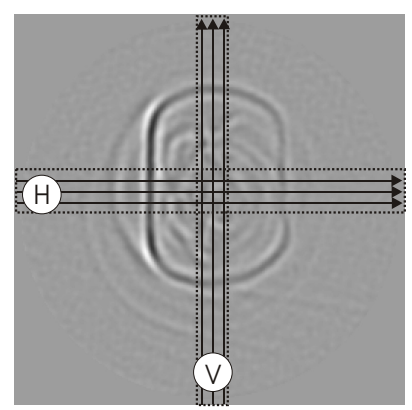

(a)

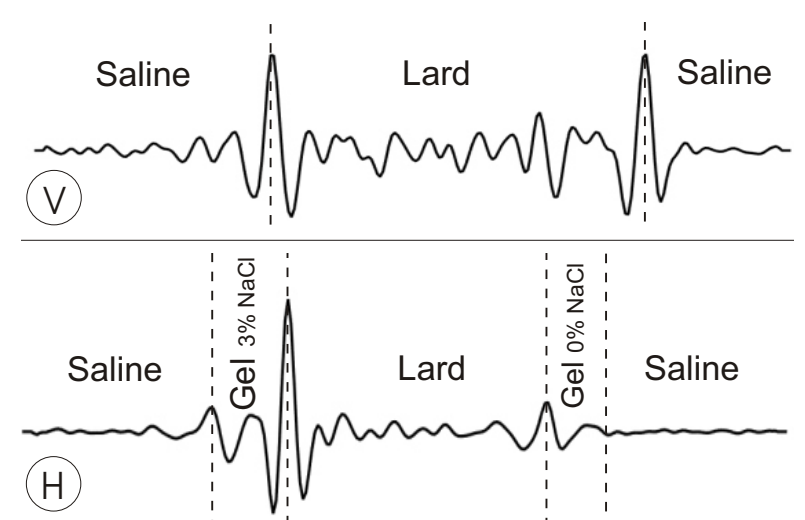

(b)

Figure 5: Average intensity profiles through the reconstructed image (a) location of rectangular regions supporting the profiles (b) profiles corresponding to the rectangles marked by letters $\mathrm{H}$ and $\mathrm{V}$ in part (a)

Lard phantom. The first phantom we present is a lard cylinder, $28 \mathrm{~mm}$ in diameter, shown in Figure 3(a) mounted on the turntable. The cylinder was intentionally mounted in an off-center position, to demonstrate that we do not take advantage of the radial symmetry of the object. The lard is practically non-conductive, yielding a very high electric contrast with the surrounding saline. The reconstruction representing a high-frequency approximation of $-\Delta \ln \sigma(x)$, is shown in Figure 3(b) as a color image and in Figure 3(c) using a gray scale. (The size of the reconstruction square (here and below) is approximately $64 \times 64$ $\mathrm{mm}$.) Figure 3(d) demonstrates intensity profile of the image along the vertical line shown in part (c). The boundary of the cylinder is clearly visible in the images. The absence of the lower frequencies leads to oscillations in the reconstruction. This is clearly seen in Figure 3(b): the boundary is represented by two yellow/red circular contours (depicting positive values) and a blue circle (showing negative values). The same oscillations are also clearly visible in Figure 3(d).

Layered phantom. Our next phantom is a cylinder consisting of several layers of different materials, shown in Figures 4(a),(b). The middle layer of the phantom consists of (non-conductive) lard. The red layer is made of agarose gel containing $3 \%$ salt $(\mathrm{NaCl})$. The conductivity of this material is quite close to the $3 \%$ saline, i.e. it is significantly higher than the conductivity of the surrounding $0.9 \%$ saline. The blue layer is also made of agarose gel without adding any salt; its conductivity is close to that of tap water, i.e. significantly lower than that of surrounding saline, but higher than that of lard. A significant drawback of agarose gel as a material for MAET phantoms is that it is water-based. This leads to a quick diffusion of the salt contained in the gel, which makes the electrical interface between the gel and surrounding saline blurred, and destroys sharp contrast we seek for our experiment. Such a diffusion was noted in the recent work on MAT-MI [14], where the authors used thin film to separate conductive and non-conductive gels. While such a technique is acceptable in MAT-MI, in MAET an application of a dielectric film would create an artificial dielectric boundary producing a strong signal of its own. We, therefore, cannot utilize the film, and 
can only remain aware of this effect.

The reconstructed images (representing $-\Delta \ln \sigma * \Xi(x)$ ) are shown in Figure 4(c),(d). Figure 5 presents the intensity profiles through the image. Since the image is noisy, in Figure 5(b) we demonstrate the average horizontal profile over the rectangle marked by letter $\mathrm{H}$ in part (a), and the average vertical profile over the rectangle marked by letter $\mathrm{V}$.

As one would expect, the most visible boundary in Figures 4 and 5 is that between nonconductive lard and highly conductive red gel. The boundary between red gel and saline is less visible, partially (we believe) due to the above-mentioned dissolution of the gel/saline interface, and partially due to lower contrast of the conductivities. The boundary between the non-conductive gel and saline is almost invisible; it is weaker than reconstruction artifacts present in the image.

Bovine sample. The third object we imaged was a beef sample containing both muscle and fatty tissues. The sample is demonstrated in Figure 6(a); it was placed in the holder as shown in Figure 6(b), with the slit tightly stitched together to avoid creating an additional jump in conductivity. The size of the sample can be estimated by comparing it to the diameter of the holder $(38 \mathrm{~mm})$. The thickness of the sample (in the vertical direction was $25 \mathrm{~mm}$. The reconstructed image is presented in Figure 6(c). The outer boundary of the sample is clearly seen in the image, with the interface between the non-conductive fat and the saline visible the best due to the high electrical contrast between these materials. The bright dot in the middle of the image corresponds to the plastic axis of the holder. This axis was located at the end of the slit; however, there is no line in the location of the slit (as intended). In order to better understand the nature of other details in the reconstruction we slit the sample horizontally. The comparison between the reconstruction and the sliced sample can be done with the help of Figure 7(a) and (b). The yellow arrows in these images highlight the boundary between the fat and muscle; inside the sample it has slightly different shape than that suggested by the image in Figure 6(a). The blue arrows highlight two lines inside the sample clearly visible in the reconstruction: they, apparently, are produced by the narrow slivers of connecting tissue visible in Figure 7(b).

\section{Conclusions and further remarks}

We have presented the 2D prototype MAET scanner, described its theoretical foundations, and demonstrated the first experimental results obtained using the scanner and the linearized reconstruction technique. One of the novel features of our scanner is the use of two pairs of electrodes, allowing for a simpler image reconstruction algorithm. While the advantage of

having multiple electrodes was demonstrated theoretically in [12], all existing experimental implementations of MAET have used one pair of electrodes.

Another important innovation in our scanner is the rotation of the investigated object, which allows us to obtain a uniformly good reconstruction of material interfaces independently of their orientation. In order to permit such a free rotation, the electrical contact with the object is implemented by the electrodes submerged in the surrounding saline, rather than by attaching them to the object. This novel data acquisition scheme, in turn, required new reconstruction techniques. We, thus, developed the theory of the 2D MAET reconstruction with point electrodes submerged in the saline and with the use of a synthetic flat transducer 


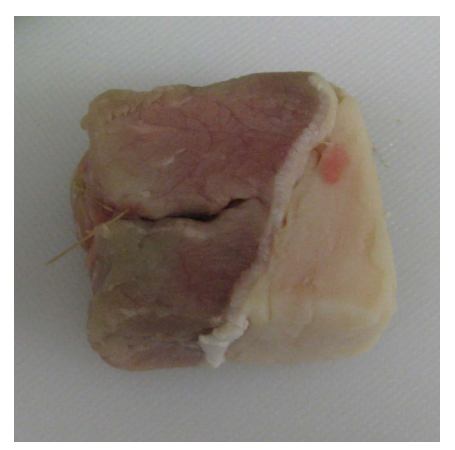

(a)

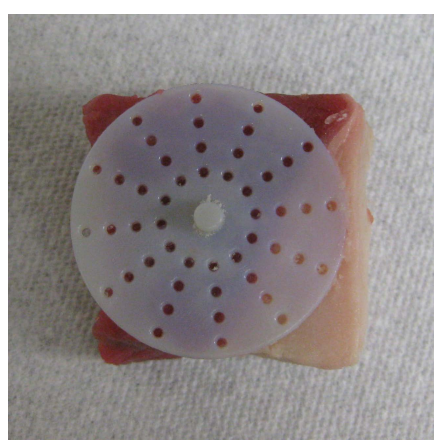

(b)

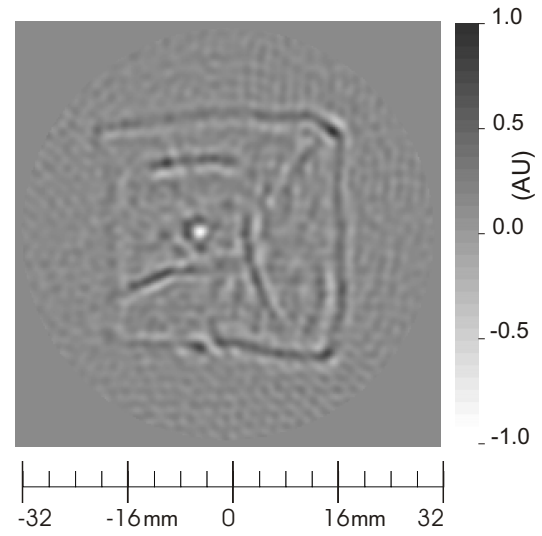

(c)

Figure 6: A meat sample and the reconstruction (a) the sample (b) sample placed in the holder (c) grey scale reconstruction

and synthetic lead currents. The more general part of the theory is presented in Section 4 (the mathematics here is discussed on an engineering level; a more rigorous study can be found in the companion paper [13]). In that section, as in all existing theoretical papers on MAET, we assumed that the acoustic excitation is wide-band, i.e. the signal contains both very low and high frequencies. Under this assumption, theoretically accurate reconstructions can be obtained using our technique, if the number of electrodes is sufficiently large.

When MAET is implemented using a piezoelectric transducer, a significant portion of lower frequencies is absent in the acoustic pulse. In this case the assumption of a wideband excitation is no longer valid, making all existing theoretical studies inapplicable. In particular, this makes impossible a qualitatively correct reconstruction of the lead currents which is a required step in [12] or in the method of Section 4. This represents a serious challenge to quantitatively accurate reconstruction of conductivity $\sigma(x)$ in MAET. For the purposes of this paper, we decided to forego such an accurate reconstruction and, in Section 5 we developed a simplified, linearized version of the reconstruction procedure, where the lead currents are assumed approximately uniform (up to a small perturbation) and known. This algorithm yields approximate reconstruction of $\Delta \ln \sigma(x) * \Xi(x)$, where $\Xi(x)$ is determined by the bandwidth of the transducer. This technique shows the boundaries between the regions with different conductivities, and/or small details whose conductivities are different from that of the surrounding medium (such as, for example, the plastic post clearly visible as the white dot in Figure 6(c)).

Interestingly, a similar effect was observed in the recent works on MAT-MI [8, 15], where only the boundaries of the regions with contrasting conductivities were also clearly visible in the reconstruction. In the latter modality the piezoelectric transducer is used in a receiving mode. However, the absence of low frequencies in the received signal has the same effect on the image as in MAET.

At least a couple of ways can be suggested for of overcoming this drawback in MAET. The first approach consists in the use of wideband acoustic sources. So far all the exper- 


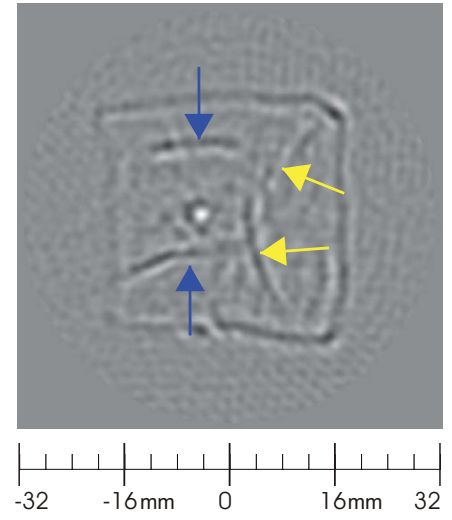

(a)

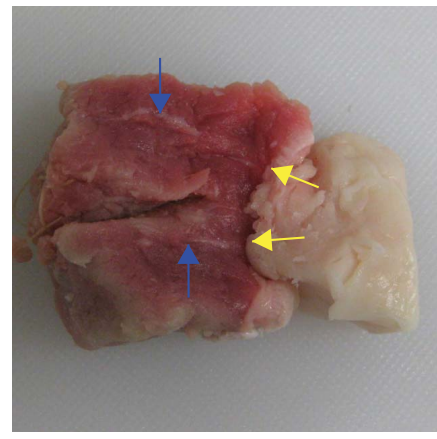

(b)

Figure 7: Comparison of the reconstructed image of the meat sample and the horizontal cut (cross-section) of the sample

imental work on MAET and MAT-MI was done using off-the-shelf diagnostic piezoelectric transducers. Instead, one could try to use custom made transducers with a wider bandwidth, or to use several transducers with different central frequencies in succesion. Alternatively, wideband acoustical pulses can be generated photoacoustically (see, e.g. [23]). Similarly, for MAT-MI one could try optical (interferometric) registration of the ultrasound signal, as it is done in photoacoustic tomography (e.g. [19]).

Another possible approach is to use available a priori information in combintion with nonlinear reconstruction algorithms to compensate for the absence of low spatial frequencies. For example, if the object is known to consist of regions with constant conductivities, methods based on total variation regularization [21] might improve the image. Feasibilty and practical usefulness of such techniques requires further investigation.

Practical results of reconstruction using our present setup were demonstrated in Section 6. They show that using the present prototype 2D MAET scanner in combination with our image reconstruction algorithm, one can image vertical boundaries of the regions with contrasting conductivities. The boundaries are recovered uniformly well, independently of their orientation, both in tissue mimicking phantoms and in a bovine sample.

A significant and well-known issue that prevents wide practical use of EIT is a severe loss of resolution in the center of the measured field. Hybrid imaging techniques, such as, in particular, MAET and MAT-MI, overcome this difficulty by using utrasound waves to extract high-resolution spatial information about the object of interest. Since utrasound pulses can propagate quite deep into soft tissues without losing the intensity or coherence, the loss of sensitivity toward the center of the object is minimal ${ }^{2}$. The results of the present paper confirm this.

We would like to view the present work as one of the first steps toward the development of the fully 3D MAET scanner capable of a quantitatively accurate reconstruction of con-

\footnotetext{
${ }^{2}$ On the other hand, presence of strong acoustic scatterers or absorbers (such as, for example, bone tissues) is likely to have a significant adverse effect on the image.
} 
ductivity in the tissues. The promising directions of the further research, in our opinion, are:

- the use of stronger magnetic fields to improve SNR;

- experimentation with alternative acoustic sources capable of wide band excitation;

- the development of alternative nonlinear procedures yielding accurate reconstruction of conductivity from MAET data obtained using existing piezoelectric transducers;

- the design of a fully 3D MAET scanner.

Acknowledgement: The authors would like to thank the anonymous referees whose comments and suggestions helped to significantly improve this paper. The work of all three authors was partially supported by the BIO5 fellowship BIO5FLW2014-04 and by NIH award NINDS R24MH109060. In addition, the first author was partially supported by the NSF awards NSF/DMS-1211521 and NSF/DMS-1418772. The authors gratefully acknowledge the use of the Rapid Prototype Printer Facilities in the Center for Gamma Ray Imaging.

\section{Appendix}

In this Appendix we explain our model of the lead current $w_{\mathbf{W}}$ given by equations (9)-(11). Within the punctured domain $\Omega \backslash \bigcup_{j=1}^{N} y_{j}$, function $w_{\mathbf{W}}(x)$ satisfies the standard conductivity equation (9), and the usual Neumann boundary condition (10) is satisfied on the nonconductive boundary $\partial \Omega$. However, at the vicinity of a point $y_{j}$ the potential must become singular, since a finite current is flowing through a point whose length is zero. Therefore, standard Dirichlet or Neumann boundary conditions cannot be prescribed at such points. Instead, we will prescribe the asymptotic behavior of $w_{\mathbf{W}}(x)$ near these points, as explained below.

Consider, for simplicity, a one-point electrode immersed in the conductive medium at $x=0$, with a current $W$ flowing into the medium. Let us assume that in a small disk $D$ (of radius $d$ ) surrounding the electrode the conductivity is constant and equal to $\sigma_{0}$ (as is the case with electrodes in our problem). Then in the punctured disk $D \backslash\{0\}$ the corresponding potential $u$ satisfies the equation

$$
\nabla \cdot \sigma_{0} \nabla u=\sigma_{0} \Delta u=0
$$

i.e., $u$ solves the Laplace equation and is a harmonic function. In a punctured disk, a general solution of the Laplace equation can be obtained by the standard technique of separation of variables in the polar coordinates $r$ and $\theta$, yielding

$$
u(r, \theta)=a_{0} \ln r+\sum_{\substack{k=-\infty \\ k \neq 0}}^{\infty} a_{k} \frac{e^{i k \theta}}{r^{|k|}}+\sum_{k=-\infty}^{\infty} b_{k} r^{|k|} e^{i k \theta}, \quad r \in(0, d), \quad \theta \in[0,2 \pi] .
$$


The total current $W$ through the (outer) boundary $\partial D$ of $D$ can be easily computed:

$$
W=\int_{\partial D} \sigma_{0} \nabla u(x) \cdot n(x) d l(x)=\sigma_{0} \int_{0}^{2 \pi} \frac{\partial}{\partial r} u(r, \theta) d \theta=\sigma_{0} \int_{0}^{2 \pi} \frac{\partial}{\partial r}\left(a_{0} \ln r\right) d \theta=2 \pi a_{0} \sigma_{0},
$$

where $n$ is the exterior normal to $\partial D$ and $d l(x)$ is the arclength. This immediately yields the needed value for the coefficient $a_{0}$

$$
a_{0}=\frac{W}{2 \pi \sigma_{0}} .
$$

The last sum in equation (40) describes the part of the solution $u^{\text {harmonic }}(r, \theta)$ that is harmonic in the whole $D$, including the origin. It is bounded by its maximum and minimum values attained somewhere on the boundary $\partial D$.

The singular behavior of $u(r, \theta)$ for small $r$ is dominated by the terms $a_{k} \frac{e^{i k \theta}}{r^{|k|}}$ with largest values of $|k|$ (if any). This implies that in our problem all $a_{k}$ except $a_{0}$ should be set to zero, otherwise the solution will be non-physical. (For example, the real part of the term $\frac{e^{i k \theta}}{r^{|k|}}$ (equal to $\frac{\cos (k \theta)}{r^{|k|}}$ ) describes a current flow that is directed toward the electrodes at those angles $\theta$ where $\cos (k \theta)$ is positive, and away from the electrode for $\theta$ with negative $\cos (k \theta))$. Therefore,

$$
u(r, \theta)=\frac{W}{2 \pi \sigma_{0}} \ln r+u^{\text {harmonic }}(r, \theta), \quad r \in(0, d), \quad \theta \in[0,2 \pi] .
$$

Correspondingly, the singular behavior of $w_{\mathbf{W}}(x)$ that is physically meaningful and yields the correct currents through the electrodes can be described by the equation (11). The fact that equations (9)-(11) lead to a solvable model is established by equations (12)-(15) that present such a solution.

Finally, one needs to verify that by imposing conditions (10) and (11) we guarantee the uniqueness of the solution up to an additive constant term (unless an additional condition is imposed, potentials are only defined up to a constant). Suppose $w_{\mathbf{W}}^{(1)}(x)$ and $w_{\mathbf{W}}^{(2)}(x)$ are two solutions of equation (9) satisfying conditions (10) and (11). Clearly, the difference $v(x) \equiv$ $w_{\mathbf{W}}^{(2)}(x)-w_{\mathbf{W}}^{(1)}(x)$ satisfies Neumann condition (10) on $\partial \Omega$ and equation (9) in the punctured domain $\Omega \backslash \bigcup_{j=1}^{N} y_{j}$. In the vicinity of points $y_{j}$ function $v(x)$ remains bounded. Therefore, as our analysis of equation (40) shows, $v(x)$ is actually harmonic in the vicinity of these points including the points themselves. Therefore, $v(x)$ satisfies the conductivity equation in the whole of $\Omega$, subject to the zero Neumann boundary conditions on $\partial \Omega$. Any constant $v(x)$ is a solution of such an equation; but it is well known that the are no other solutions (see [16], Thm 5.IV). Therefore, $w_{\mathbf{W}}^{(2)}(x)$ and $w_{\mathbf{W}}^{(1)}(x)$ can differ only by a constant. Thus, our model of the lead potential $w_{\mathbf{W}}$ represented by equations (9)-(11) has a unique solution (up to an additive constant term). Correspondingly, currents $\mathbf{J}^{\mathbf{W}, \sigma}$ are defined by equation (16) uniquely. 


\section{References}

[1] H. Ammari, P. Grasland-Mongrain, P. Millien, L. Seppecher and J.-K. Seo, A mathematical and numerical framework for ultrasonically-induced Lorentz force electrical impedance tomography J. Math.Pures Appl. 103(6) (2015) 1390-1409.

[2] D. C. Barber, B. H. Brown, Applied potential tomography, J. Phys. E.: Sci. Instrum. 17 (1984), 723-733.

[3] L. Borcea, Electrical impedance tomography, Inverse Problems 18 (2002) R99-R136.

[4] M. Cheney, D. Isaacson, and J. C. Newell, Electrical Impedance Tomography, SIAM Review, 41, (1999) 85-101.D.

[5] Colton and R. Kress, Inverse acoustic and electromagnetic scattering theory, SpringerVerlag (2001).

[6] P. Grasland-Mongrain , J.-M. Mari, J.-Y. Chapelon, and C. Lafon, Lorentz force electrical impedance tomography IRBM 34 (2013) 357-60.

[7] S. Haider, A. Hrbek, and Y. Xu, Magneto-acousto-electrical tomography: a potential method for imaging current density and electrical impedance, Physiol. Meas. 29 (2008) S41-S50.

[8] G. Hu, E. Cressman and B. He, Magnetoacoustic imaging of human liver tumor with magnetic induction Appl. Phys. Letters 98 (2011) 023703.

[9] P. Kuchment and L. Kunyansky, Mathematics of Photoacoustic and Thermoacoustic Tomography, Chapter 19, Handbook of Mathematical Methods in Imaging, SpringerVerlag, (2011) 819-65.

[10] P. Kuchment and L. Kunyansky, Synthetic focusing in ultrasound modulated tomography, Inverse Problems and Imaging, 4 (2010) 665-73.

[11] P. Kuchment and L. Kunyansky, 2D and 3D reconstructions in acousto-electric tomography, Inverse Problems 27 (2011) 055013.

[12] L. Kunyansky, A mathematical model and inversion procedure for Magneto-AcoustoElectric Tomography (MAET) Inverse Problems 28 (2012) 035002

[13] L. Kunyansky, C. P. Ingram and R. S. Witte, Rotational Magneto-Acousto-Electric Tomography (MAET): mathematical foundations, in preparation.

[14] X. Li, Y. Xu and B. He, Imaging electrical impedance from acoustic measurements by means of Magnetoacoustic Tomography With Magnetic Induction (MAT-MI), IEEE Trans. Biomed. Eng, 54, (2007) 323-30

[15] L. Mariappan and B. He, Magnetoacoustic tomography with magnetic induction: bioimepedance reconstruction through vector source imaging, IEEE Trans. Med. Imag. 32(3), (2003) 619-27. 
[16] C. Miranda (1970) Partial differential equations of elliptic type, 2-nd edition, Berlin, Springer-Verlag.

[17] A. Montalibet, J. Jossinet, A. Matias, and D. Cathignol, Electric current generated by ultrasonically induced Lorentz force in biological media, Med. Biol. Eng. Comput. 39 (2001) 15-20.

[18] F. Natterer (1986) The mathematics of computerized tomography, New York, Wiley

[19] G. Paltauf, R. Nuster, M. Haltmeier, and P. Burgholzer, Photoacoustic tomography using a Mach-Zehnder interferometer as an acoustic line detector, Appl. Opt. 46(16) (2007) 3352-8.

[20] B. J. Roth and K. Schalte, Ultrasonically-induced Lorentz force tomography, Med. Biol. Eng. Comput. 47 (2009) 573-7

[21] L. I. Rudin, S. Osher and E. Fatemi, Nonlinear total variation based noise removal algorithms, Physica D 60 (1992) 259-68.

[22] H. Wen, J. Shah, R. S. Balaban, Hall effect imaging, IEEE Trans. Biomed. Eng., 45 (1998) 119-24.

[23] G. Wurzinger, R. Nuster, N. Schmitner, S. Gratt, D. Meyer,and G. Paltauf, Simultaneous three-dimensional photoacoustic and laser-ultrasound tomography, Biomed. Optics Express 4(8) (2013) 1380-9.

[24] Y. Xu and B. He, Magnetoacoustic tomography with magnetic induction (MAT-MI), Phys. Med. Biol., 50 (2005) 5175-87

[25] R. Zengin and N. G. Gençer, Lorentz force electrical impedance tomography using magnetic field measurements, Phys.Med.Biol. 61 (2016) 5887-905 